\title{
Simulation and Control of Turbulence at Tokamaks with Artificial Intelligence Methods
}

\author{
Danilo Rastovic \\ Control Systems Group, Zagreb, Croatia \\ Email: dr.rastovic@excite.com
}

Received August 11, 2012; revised October 18, 2012; accepted October 28, 2012

\begin{abstract}
The control of turbulence at tokamaks is very complex problem. The idea is to apply the fuzzy Markovian processes and fuzzy Brownian motions as good approximation of general robust drift kinetic equation. It is obtained by using the artificial neural networks for solving of appropriate advanced control problem. The proof of the appropriate theorem is shown.
\end{abstract}

Keywords: Tokamak; Turbulence; Control; Artificial Intelligence; Drift Kinetic Equation

\section{Introduction}

High- $\beta$ regimes are difficult to operate in, mostly because of macroscopic instabilities leading to the disruption of the plasma column and termination of discharge. Whether driven by magnetic of electrostatic turbulence, anomalous transport causes a large increase in the energy losses, and poses severe constraint on the amount of auxiliary power required to heat up the plasma to the high temperatures required for thermonuclear ignition.

When the plasma rotates either toroidally or poloidally, both the energy transport as well as the macroscopic stability improve significantly. The plasma rotation can be either spontaneous or driven by neutral beam injection or radio frequency (RF) heating.

Flow modified equilibria will almost certainly exibit stability properties different from the static equilibria, which need to be investigated with new kinds of nonvariational stability codes. Flow shear (both toroidal and poloidal) is found to be stabilized, while the bulk toroidal rotation can be either stabilizing or destabilizing.

Turbulence in plasmas is an ever present theme in plasma physics, including in reactor-size tokamaks. An obvious way to begin a study of the kinetics would be to use an experimentally measured turbulence potential. The second possible way is to simulate the generation of the turbulence by a given system of equations. We can consider all possible types of motion: regular motion, Gaussian diffusion and Levy walk diffusion.

Pure averaging prevents one from recognizing important effects, in particular the effect of "islands around islands". This type of potential region, which separates regular motion from the "stochastic sea", is considered by Zaslavsky et al. [1] as the real seed of anomalous diffusion. The problem is how to synchronize all these effects by the methods of artificial intelligence.

Using the advantage of using the Poincare surfaceof-sections, we can track the changes in the dynamics of particles as the initial conditions approach the threshold between regular motion and a stochastic sea (the stickness effect), as well as the fractality of the kinetics.

In view of significance of the control of delayed neural networks, in recent years, the stabilization and synchronization of neural networks with delays have been extensively investigated by many researchers [2]. As is widely known, noises do exist in a neural network due to random fluctuations and probabilistic causes in the network. Thus, it is necessary and rewarding to study stochastic effects to the dynamic behaviours of neural networks [3].

Operations with high- $\beta$ plasma are advantageous in the tokamak reactor from the economical point of view. The high- $\beta$ operations mean a high fusion output even with a compact device, that is, the operation can be said to be economically efficient. On realistic devices, the stabilizing effect is lost owing to the wall resistivity since a conducting wall has a finite resistivity. The finite resistivity of the conducting wall induces another instability, that is, the resistive wall mode (RWM).

Since the RWM is thought to limit the achievable $\beta$ in a realistic device, RWM stabilization is one of the key issues for ITER and reactors. Theoretically, plasma rotation suggested to strongly affect the RWM stability.

Experimentally, plasma rotation stabilization has been observed. For understanding the stabilization with a slow plasma rotation, kinetic effects of bulk plasma and fast 
ions have been taken into account. From these reasons we will apply advanced variational calculus which include also the applications of artificial neural networks in the process of learning during optimization of results.

\section{Tokamak Experiments}

For more than 30 years fusion researchers have sought to understand a variety of abrupt transitions in plasma operating conditions observed in tokamak experiments, and to anticipate others that might be present in fusion reactors, by analyzing instabilites in the coupled plasma particle, momentum and energy balance-i.e. thermal instabilities.

A strong temperature dependence of energy transport loss rate has been identified a driver or a stabilizer of thermal instabilities, depending on the sign of the temperature dependence. Many of these drivers of thermal instabilities are primary edge phenomena. Because the onset of the edge-related thermal instabilities can be theoretically related to local or average densities exceeding a threshold value, density limits for the onset of these observed phenomena can be related to the onset of the respective thermal instabilities. The onset conditions are determined by a balance between destabilizing termimpurity and the stabilizing term-conduction.

The temperature dependence of the threshold densities is more complex, because of the very different temperature dependence of the various stabilizing and destabilizing terms.

The thermal instability explanation of the L-H and $\mathrm{H}-\mathrm{L}$ transitions and of observed confinement degradation, while supported by experimental comparations, is new, and there exists a much larger body of competing explanations for these particular experimental phenomena.

Looking ahead to burning plasmas, the role of the destabilizing temperature dependence of the fusion cross section is well understood, several active control mechanisms have been investigated, and the possibility of inherent stabilization via the temperature dependence of thermal transport coefficients has been identified.

Magnetic islands and neoclassical tearing modes (NTMs) can produce sawtooth crashes and disruptions. Many of the proposed solutions are related to the employment of resonant magnetic perturbations (RMPs). A RMP is a magnetic perturbation characterized by a helical resonant inside the plasma and typically produced by a dedicated set of active coils [4]. Recently, RMPs have been utilized to produce stochasticity at the plasma edge.

We can show the equivalence of the first variation of the electrostatic entropy and the canonically averaged Lagrangian with respect to adiabatic variations. One finds equivalence between the Hamilton's action principle and the vanishing of the first variation of nonlinear electrostatic entropy with respect to adiabatic variations.

The magnetic configuration of the plasma is time independent. This is the case of the tokamak in static equilibrium. Time independent magnetic configurations of the plasma associated with a time independent magnetic entropy are called "plasma states". The electric field present in a plasma state can only be the stationary electric field induced externally.

The requirement that the magnetic entropy is stationary in the tokamak implies the balance between the net power deposited on electrons and the power lost by electron thermal conductivity. The additional requirement that the balance holds locally leads to an equation for the profile of the current density induced by external electric field (the stationary magnetic entropy equation) [5]. We can investigate how the magnetic entropy changes in time when the plasma evolves in accordance with Maxwell equations.

One can discuss the stability of the magnetic equilibrium in a variety of physical situations depending on the special form of the current density. The pressure profiles in tokamaks are restricted by the requirement that the thermal energy of the plasma be minimum for fixed magnetic entropy and fixed total current.

Chaotic systems have been exploited for applications such as control and random number generation. In these applications, it is desirable that the underlying chaotic attractor is robust so that random fluctuations or small disturbances would not cause the system to transit to a periodic state.

There are many problems faced, including the effect of injection heating and the different fuelling scenarios for power and particle balance of fusion plasma. We can consider not only fluctuations but also modify the time variation of particle fuelling scenarios. It is shown that a slow variation of the helium ash density with time can be used for operations path-changing in deuterium-tritium fusion plasma. The main target of the study is the optimization of the plasma operation scenario in future fusion reactors including ITER.

The evolution of equilibrium was considered in connection with the diffusion of magnetic fluxes and the redistribution of the current, without taking account of the evolution of the pressure. Here attention is concentrate here on the correlation of the evolution of equilibrium and heat transfer, and the current distribution is taken to be specified.

The attainment of thermonuclear regimes, with high temperature, very long resistive diffusion time scales, high $\beta_{p}$ effects, is finally dependent on major problem of tokamak physics and operation, namely the control of the inevitable instability of slow-growing resistive modes. In many cases actual plasmas are far from thermal equilibrium, being inhomogeneous or having non-Maxwel- 
lian velocity distributions, features which significantly complicate the analysis of experimental data. In addition, these departures from thermal equilibrium can trigger instabilities, whose growth and non-linear saturation eventually lead to the onset of turbulence.

The power of the thermonuclear burning will be stabilized at a fixed level by automatic regulation of the power of additional heating. In this case the reactor operates in the subcritical regime, i.e., the parameter of the plasma are chosen so that the power of the thermonuclear reactions is slightly less than the power lost. This difference is compensated by additional heating, the power of which follows the thermonuclear power via negative feedback.

It is obvious that as the thermonuclear power increases feedback will increase the additional heating power. Therefore the installation must be provided with reserve power capable of compensating the oscillations of $S_{\alpha}$ for $\alpha$-particles. The value of this reserve power depends on the parameters of the plasma and the technically determined desirable accuracy of the power regulation.

In fusion reactor design, a radiative divertor concept is widely adopted to suppress erosion at the divertor plates and to reduce the localized heat load on the divertor plates.

\section{Tokamaks and Turbulence}

In the coming year we plan to contribute broadly to theoretical modelling of ITER-like and spherical tokamaks. The range of topics and activities envisaged is listed below:

- Contribute to projects such as equilibrium reconstruction, fast particle physics and turbulence code benchmarking.

- Develop and implement the fully parallelised version of global two-fluid turbulence simulation code with demonstration applications to MAST and other tokamaks.

- Use the local gyro-kinetic code for modelling microinstabilities in MAST.

- Continue the modelling of the effect of tearing mode islands on fast particle confinement in spherical tokamak geometry with application to MAST.

Understanding the role of turbulent processes in magnetically confined plasmas is central to understanding their transport and confinement properties.

We can analyze turbulent electrostatic field in a stationary toroidal magnetoplasma, created by radio-frequency waves and confined by two different toroidal magnetic fields. Observations suggest that turbulence has recurrent properties, as those observed in recurrent fully chaotic low-dimensional systems. Therefore, evolution of measurements of low-dimensional systems can be used to describe the recurrence observed in the tokamak edge turbulence.

The investigation of the correlations between, on the one hand, the occurence of transport barriers and improved confinement in magnetically confined plasmas, and on the other hand, electric fields, modifed magnetic shear and electrostatic and magnetic turbulent fluctuations necessitates the use of various active means to externally control plasma transport. It also requires one to characterize fluctuations of various transport barriers and to elucidate the role of turbulence driving and damping mechanisms, including the role of the plasma edge properties.

There are some conventional control theory methods of the stabilization of the resistive wall mode (RWM) instability at tokamaks [6]. Our aim is the application of the conventional control theory together with the methods of fuzzy neural control theory.

The most challenging aspect of the H-mode physics is the sudden disappearance of the turbulence whereas its driving forces - the gradients at the plasma edge-increase. The turbulence, which normally limits the confinement, induces a sheared plasma flow, which acts back to its generating origin and quenches the fluctuations. One part of this process-turbulence produces zonal flows via Reynolds stress plays a role in normal fluids also.

The turbulence tends to drive large scale flows, which are in turn mitigated through interaction with toroidal magnetic equilibrium. The understanding of turbulent transport processes which govern the energy, momentum and current distributions in tokamak plasmas is important tool to optimizing the economically viable design of future power plants based on tokamak concept. Zonal flows can lead to substantial reduction of turbulent transport in localized region as transport barriers.

Understanding and predicting turbulent transport is a key issue on the way toward commercially, viable fusion reactors. Indeed turbulence control the confinement control of any magnetically confined plasma.

Obviously, gyrokinetic theory is intimately entangled in the physics of turbulent transport in magnetized fusion plasmas. Zonal flows are generated by turbulence and back react on turbulence via vortex shearing and convection from locally stable to unstable regions. We obtain a familiar expression of the local gyrokinetic equation

$$
\begin{aligned}
& \frac{\partial}{\partial t}+v_{\|} b \cdot \nabla+\frac{c}{e_{s}} \frac{1}{B_{0}} b \times\left(e_{s}(\psi)_{\xi}+m_{s} v_{\|}^{2} b \cdot \nabla b+\mu \nabla B_{0}\right) h_{s} \\
& =C_{G Y}\left(\delta f_{s}\right)-\left(e_{s} \frac{\partial \psi_{\xi}}{\partial t} \cdot \frac{\partial f_{M S}}{\partial \varepsilon}+\frac{c}{B_{0}} b \times \nabla \psi_{\xi} \cdot \nabla f_{M S}\right)
\end{aligned}
$$

where $\delta f_{s}=\delta H_{s} \partial f_{M S} / \partial \varepsilon+h_{s}$.

This model is an open system developed to study local quasi-steady turbulent transport with fixed background 
profiles. Although the conservation properties in the original gyrokinetic equations are lost, the quality of the nonlinear simulation is often tested using the entropy balance relation [7].

Domain decomposition is in principle applicable to the all three classes of schemes (Lagrangian, Eulerian and semi-Lagrangian). The geometry of the magnetic configuration strongly effects microinstabilities and turbulence. The idea is the application of fuzzy neural simulations of the Vlasov-Poisson-Fokker-Planck (VPFP) equation to obtain some kind of variational calculus for the plasma confinement problem in fusion magnetic devices. The stochastic part of kinetic equation is described with Brownian motion. The Markov processes are good approximations of Brownian motion. In tokamak physics is necessary work with interval numbers as uncertainities. We can interpret these intervals with words. In this case we obtain fuzzy Markovian processes and fuzzy Brownian motions. The new changes are incorporated in kinetic plasma equation as fuzzy neural networks.

Non-equilibrium turbulent processes are anisotropic, non-local, multi-scale and multi-phase, and often are driven by shocks or acceleration. Their scaling, spectral and invariant properties differ substantially from those of classical Kolmogorov turbulence. At atomistic and mesoscales, the non-equilibrium dynamics depart dramatically from a standard scenario given by Gibbs statistical ensemble average and quasi-static Boltzmann equation.

At dissipative scales, where the fluid flow is differentiable, the phase-space density of particles is supported on a dynamically evolving fractal set. This attractor is characterized by non-trivial multiscaling properties.

Description of transition from regular to random regimes of motion and characterization of the intermediate states where both regular and random wave motions are present and mutual inter-connected, is an intriguing and challenging problem. Recent technical progress allows to investigate, both experimentally and numerically, the correlation properties of fully developed turbulence in terms of particle trajectories.

It has recently become possible to compute precise equilibrium, traveling wave, and periodic orbit solution above the onset of turbulence. These invariant solutions capture the complex dynamics of unstable coherent structures in wall-bounded flows and provide a framework for understanding a turbulent flows as dynamical systems. We present a number of weakly unstable equilibria and periodic orbits and visualizations of their physical and state-space dynamics. Applications could be found in magnetic confinement fusion.

The understanding and reduction of turbulence transport in magnetic confinement devices is not only an academic task but also a matter of practical interest, since high confinement has been chosen as the regime for
ITER and possible future reactors because it reduces size and cost. Turbulence comes in two classes: electrostatic and magnetic turbulence. Turbulence can be externally controlled, which led to better and better confinement. Electrostatic turbulence stabilization concept has the universality, needed to explain ion transport barriers at different radii seen in limiter and divertor tokamaks with a variety of edge biasing schemes. Magnetic turbulence drives the anomalous electron heat conduction [8].

Turbulence plays a very important role in particles and energy cross-field transport to the wall in the edge plasma. The problem is extract the maximum of information from data. Taking into account the intrinsic intermittent and nonlinear character of turbulent data, the Fourier methods are not well suited. The useful can be the advanteges of the wavelet techniques and how these techniques make it to resolve problem of quantitative characterization of intermittency and of the self-similarity properties of turbulence and of space-time characterization by measuring correlations between data taken from different locations.

Theoretical description of non-equilibrium transports is a challeging problem due to singular aspects of the governing equations. Furthemore these processes are stochastically unsteady and their fluctuating quantities are essentially time-dependent and non-Gaussian. It is shown that their invariant, spectral, scaling and statistical properties differ substantially from those of isotropic homogeneous turbulence.

\section{Approach with Control Theory}

Some characteristics of transport in fusion plasmas indicate that it may be a manifestation of a self-organized and critical system (SOC). These characteristics are: existence of critical gradients, the existence of transport events that do not obey diffusive equations and global scaling. If a magnetically confined plasma is a SOC system it might lead to novel ways of controlling turbulence.

The Hurst coefficient is closely related to the decay of the auto-correlation function at long lags and implies the existence of long-range time correlations. The range of values of $H$ in the edge plasma is between 0.6 and 0.74 but in the scrape-off-layer (SOL) displays a much larger variation, between 0.5 and 1 .

At W7-AS, a radial profile of $H$ was obtained that proved to be reproducible in various similar discharges having a maximum near separatrix [9]. The process of autonomous convergence towards an ordered complex form, also called self-organization process, requires form that have already reached a somewhat advanced stage in ordering process. For example, in nonlinear thermodynamics this principle will be called self-organization. In the selforganization theory-a convergence towards the 
maximum interaction is obtained (positive feedback [10]).

High-power radio frequency in the ion-cyclotron range of frequencies (ICRF) have the potential to heat and control the ITER plasma through localized energy deposition, driven current, and driven plasma flows. FokkerPlanck coupled radio-frequency simulations show that because of the high plasma density, energetic ion tail formation in ITER is typically weak, with the expection of the minority deuterium heating scheme where strong tails can develop on the minority ion distribution.

Control of plasma density and temperature magnitudes, as well as their profiles, are among the most fundamental problems in fusion reactors. In thermally unstable zone, and active control system is necessary to stabilize the thermonuclear reactions. The controller makes use simultaneously of the modulation of auxiliary power, the modulation of fueling rate and the controlled injection of impurities as actuators. Radiative divertor conditions were obtained by injection of gaseous impurities into divertor.

When the dynamics within a scattering region is chaotic, we say that we have a chaotic scattering. Chaotic scattering is a very active research topic in dynamical systems. In analogy to more general dynamics, chaotic scattering can be hyperbolic or nonhyperbolic. Hyperbolic scattering is associated with an exponential decay of probability of particles to be found in the scattering region after a given time $t$. In the paper [11] is given scattering theory for linear transport for the case of neutrons. The exponential decay of probability for particles in the case of linear transport theory for neutrons and ions is shown in the papers $[12,13]$.

Nonhyperbolic scattering is characterized by the presence of Kolmogorov-Arnold-Moser (KAM) islands in phase space. These islands surround marginally stable periodic orbits. Nonhyperbolic escape is characterized by a power law distribution of probability [14]. This is the case of general transport theory for ions. The interesting question is when the power law converges in averaging to the exponential decay. There is mathematical interprettation of such assertion [15].

New simulation tools open the route to the investigation of plasma turbulent transport with increasingly selfconsistent interplay between various scales and transport mechanisms to drive such a complex system.

The system is governed by the following dimensionless equations:

$$
\begin{aligned}
& \frac{\partial f_{G}}{\partial t}=-\left(\boldsymbol{v}_{E}+\boldsymbol{v}_{g}+\boldsymbol{v}_{G \|}\right) \cdot \nabla f_{G}+\frac{\mathrm{d} v_{G \|}}{\mathrm{d} t} \cdot \frac{\partial f_{G}}{\partial v_{G \|}} \\
& +S+C\left(f_{G}\right)+D\left(f_{G}\right)
\end{aligned}
$$

[16]. The guiding-centre distribution function $f_{G}$ is normalized as follows: $f_{G} \rightarrow\left(n_{0} / v_{T 0}^{3}\right) f_{G}$. The transverse drifts and parallel dynamics are governed by the following set of equations:

$$
\begin{aligned}
& \boldsymbol{v}_{E}=\frac{\boldsymbol{B}}{B^{2}} \times \nabla(J \cdot \Phi) \\
& \boldsymbol{v}_{g}=\frac{1}{B}\left(v_{G \|}^{2}+\mu B\right) \cdot \frac{\boldsymbol{B}}{B^{2}} \times \nabla B \\
& \frac{\mathrm{d} v_{G \|}}{\mathrm{d} t}=-\nabla_{\|}(J \cdot \Phi)-\mu \nabla_{\|} B+v_{G \|} \boldsymbol{v}_{E} \cdot \frac{\nabla B}{B}
\end{aligned}
$$

Equation (2) conserves the three equilibrium motion invariants in the collisionless regime, namely the magnetic momentum $\mu$ (trivial), the total energy

$$
H=v_{G \|}^{2} / 2+\mu B+J \cdot \Phi(r, \theta, \varphi)
$$

and the toroidal kinetic momentum $L_{\varphi}=R v_{G \varphi}+\psi$ due to axi-symmetry (with $\psi$ the poloidal magnetic flux).

Ion temperature gradient (ITG) turbulence is often found to be main cause of anomalous transport in fusion experiments. If the plasma conditions are chosen appropriately, it is also possible to experimentally investigate "pure" trapped electron mode (TEM) regimes and compare with turbulence simulations. But in more conventional situations, the application of several heating methods (ECRH) plus neutral beam injection (NBI) and/ or a sufficiently high collisionality leads to finite (and often similar) values for the temperature gradients of all species, which usually means that several linear instabilities are present simultaneously.

The interaction between the different unstable modes at the TEM-ITG transition produces some interesting phenomena, including a supression mechanism for the particle flux. Some of these features can be understood at least qualitatively by quasilinear investigations, which offer the possibility of extended parameter scans.

The collective configuration can exchange electric charges with the medium and therefore the amplitude of the collective quantities is not fixed but fluctuates. In the case of an inhomogeneous plasma one can define an electrostatic entropy functional which is sensible to the local structure of the equilibrium. The electrostatic interaction energy has a precise physical meaning.

The simple case of the electrostatic equilibrium is described by the one-dimensional Vlasov equation, static in the laboratory frame of reference

$$
v \cdot \frac{\partial f_{s}}{\partial x}+\frac{q_{s}}{m} \cdot E(x) \cdot \frac{\partial f_{s}}{\partial v}=0
$$

An oscillatory one-dimensional static solution of the electrostatic Vlasov equation is always associated with a non-Maxwellian distribution function. It can be shown that the minumum of the electrostatic entropy corresponds to the instability of the collective equilibrium predicted by Vlasov equation. Any distribution function 
with a single maximum is stable and corresponds to the maximum of the entropy. The Maxwellian case is contained consistently in the theory.

The ultimate application of fusion power as an energy source requires not only a high fusion reaction rate but also steady-state operations, which often necessitates high plasma pressure. Unstable resistive-wall modes (RWMs) were found to have been suppressed by either rapid plasma rotation or active feedback control in various toroidal devices. To study the details of the RWM feedback process and assess the RWM feedback performance, highly reproducible Ohmic discharges with fast plasma current ramp-up unstable to the current-driven RWMs at edge safety factor have been developed in the DIII-D tokamak.

The error fields are externally imposed non-axisymmetric fields that couple to the kink mode and arise from inevitable irregularities of the magnetic configuration of the tokamak. There is a dynamically varying error field whose origin is unknown and appears only during plasma operations. From this point of view necessity is to have the real time controller in the process. Not all the feedback coil currents are directly used for suppressing the RWM. In that regard, it has been challenging to identify the partition of coil currents directly.

The direction of the toroidal rotation can be reversed when the mode frequency decreases. This provides a natural explanation for the toroidal flow reversal when plasma make a L-H transition observed in Alcator CMOD. The quasilinear theory of toroidal momentum confinement is not only applicable to Ohmic-heated plasmas, but also to radio-frequency-wave (RF) heated tokamak if there is no toroidal momentum input associated with the RF sources. In this regard, the theory may provide an explanation for the "corotation" observed in RF-heated Alcator C-MOD plasmas. One can speculate that either RF wave electric field stabilizes ion temperature gradient driven turbulence or RF heating modifies ion temperature profile and causes mode frequency to decrease. This decrease leads to "corotation". The difference in RF-induced $\boldsymbol{E} \times \boldsymbol{B}$ drift can cause a charge accumulation opposite to that of the instability. The instability could thus be stabilized. The stabilization mechanism can be due to the finite ion Larmor radius effects.

Now, after some 30 years of tests using the Alcator series of reactors, the MIT researches have found one mode of operation, which they call I-mode (for improved) on which the heat stays tightly confined but the particles, including contaminants, can leak away. When a fusion reactor operates, the impurities accumulate. The operating conditions and the control requirements to stay in I-mode need to be better undestood.

The I-mode eliminates or greatly reduces the occur- ance of unpredictable bursts of heat from the edge to the confined plasma. Depending of how the strenght and shape of the magnetic field are set, both heat and particles can constantly leak out of plasma (in a setup called Lmode, for low-confinement) or can be held more tightly in place (called H-mode, for high confinement). In Imode the heat stays tightly confined, but the particles, including contaminants, can leak away. The findings could be useful when fusion power is sustained mostly by "self-heating" and don't need large amounts of outside power.

Experimental results indicate the existence of azimuthal (toroidal) plasma rotation in tokamaks subjected to neutral beam heating. In field-reversed configurations azimuthal rotation is responsible for a type of instability that may destroy plasma confinement. One possible approach to investigate the effects of rotation on MHD equilibrium and stability properties would be to obtain numerical solutions of the 3-dimensional ideal MHD equations. Magnetic flux surfaces rotate rigidly with the plasma, according to Alfen's theorem, and are characterized by poloidal flux and current functions that satisfy an elliptic partial differential equation which, in the limit of vanishing rotation, is reduced to the Grad-Shafranov equation. For these purposes is good apply the approach with fuzzy mathematics. In this case, the fuzzy Markovian processes describe the behavior of the solution of Fokker-Planck equation, as generalization of Brownian motion, for Vlasov-Poisson-Fokker-Planck equation.

\section{The Methods of Artificial Intelligence}

Stellarator posses an equilibrium parameter space that is greather than that of tokamaks because of the full threedimensional nature of stellarator plasmas. Some disruptive instabilities nowdays can be avoided thanks to the increased experimental experience with tokamak operation, and instabilities of the plasma position are tackled with improved magnetic control techniques, for instance.

Plasmas with shaped cross sections have higher magnetohydrodynamic stability limits, but require sophisticated feedback control. Moreover, vertically elongated plasma is vertically unstable and requires feedback stabilization. We can consider the design of a Kalman filter to reconstruct the states relevant for plasma vertical stabilizator. But for general nonlinear, stochastic VlasovPoisson-Fokker-Planck systems we must use Ito calculus.

Genetic algorithms rely on the concept of natural selection mechanisms and genetic operators. The GAs is generally decoposed into the following steps:

1) Random construction of an initial population and evolution of its fitness.

2) Choice of the subset according to its fitness and breading a new population. 
3) Evaluation of the fitness of each new individual and replacement of the old population with the new one.

4) Checking whether the fitness has reached a predefined value or the iteration number (generation) has reached a certain value. If not, reiteration from step 2 .

This is the way toward application of modern advanced Monte Carlo methods.

Disruptions are sudden and unavoidable losses of confinement that may put at risk the integrity of a tokamak. However, the physical phenomena leading to disruptions are very complex and non-linear and therefore no satisfactory model has been devised so far either for their avoidance or their prediction. For this reason, machine learning techniques have been extensively pursued in the last years. Disruptions can be triggered by various instabilities which, on time scale even of the order of milliseconds, can force the plasma out of its operational limits.

Artificial neural networks and support vector machines (SVMs) are capable of building general models, by "learning" from the data in the so-called "training process". In the paper [17] the complete evolution of the considered discharges is followed from the beginning to the end to determine whether a disruption if fortcoming or not. We will try to describe the using of artificial neural networks in investigation of behavior of nonlinear VlasovPoisson-Fokker-Planck systems that describe the turbulence.

In the past decades, the fuzzy logic theory has been shown to be an effective approach to dealing with the analysis and synthesis problems of nonlinear systems. Among various models available to fuzzy systems, the Takagi-Sugeno (T-S) fuzzy model was a notible one which was a linear system constructed to approximate a nonlinear plant. Roughly speaking, the T-S fuzzy model is a system described by fuzzy IF-THEN rules which can give local linear representation of the nonlinear system by decomposing the whole input space into several partial fuzzy spaces and representing each output space with a linear equation.

We consider a generalization of the traditional Takagi-Sugeno fuzzy system that includes both the parameter uncertainities and the stochastic disturbances. The corresponding $i$ th fuzzy rule is formulated in the following form:

Plant rule $i$ :

If $\theta_{1}(t)$ is $\eta_{i 1}$ and... $\theta_{p}(t)$ is $\eta_{i p}$ then

$\mathrm{d} f(t)=$

$\left[\left(A_{i}+\Delta A_{i}\right) f(t)+\cdots+\sigma_{i}(t, f(t), f(t-\tau(t)))\right] \mathrm{d} \omega_{i}(t)$,

$\omega_{i}(t) \in R^{q}(i=1,2, \cdots, r)$ are uncorrelated zero mean Gaussian white noise processes with covariances $I_{q}$.

There been performed studies on the correlation be- tween electrostatic and magnetic fluctuations. The connection between turbulent density fluctuations and lowdimensional chaotic dynamics has been quantitatively verified by using suitable numerical algorithms like the correlation dimension and the statistical distribution of recurrence times.

One of the most difficult challenges in computational statistical physics is sampling of low-temperature equilibrium states of the systems. Standard Markov Chains Monte Carlo (MCMC) methods become stuck in local minima and are unable to correctly sample the equilibrium distribution. In parallel tempering, many replicas of the system are simultaneously simulated using a standard MCMC method with each replica at a different temperature. Replica exchanges are allowed that permit replicas to move between low and high temperatures. We must use the adaptive Monte Carlo.

Competitive learning neural networks are regarded as a powerful tool for online data clustering to represent a non-stationary probability distribution with a fixed numer of weight vectors. One difficulty in applications of competitive learning neural networks to online data clustering is that most of them require heuristically-predetermined threshold parameters of balancing a trade-off between accuracy, i.e. error minimization performance, and speed of adaptation to the changes in source statistics. Both quick adaptation and error minimization are simulteneously accomplished without any carefully predefined parameters.

When modeling transport processes in tokamak plasmas the following assumption prevails: widely separated regions of the plasma do not significantly interact with each other. This paradigm states that any particle or heat flux can accurately be described using a set of local transport coefficients.

Transport events are random and accurately described by a classical Gauss-Markov process. Nor is taken into account the fact that a large localized event may have a significant aftereffect in the same region it occured in. Any transport model should endeavor to encompass the possibility for a nonlocal, nondiffusive action at a distance. The heat flux $Q(r, t)$ has been temporally averaged over a collision time; we thus only addressed the spatial question of the nonlocal, nondiffusive (non-Gauss-Markov) behavior of $Q$. An equally question is now to address its Markov (temporal) counterpart. A strong nonlocal, nondiffusive spatial behavior is found, stremming from the selforganized transport.

At least, the available data does not allow to conclude on the possibility of non-Markovian (memory) effects. With the introducing of artificial neural networks for the non-Markovian process is transfered into Markovian process [18]. 


\section{Drift Kinetic Equations and Advanced Optimal Control}

The analysis of electrostatic fluctuations is of great interest in understanding the degradation in confinement of a magnetically confined plasma. Diagnostic methods are mostly based on digital correlation of data applying different models for their analysis. Some diagnostics are based on a statistical processing of signals.

We must identify systematically and properly the kind of instability responsible for the turbulence and the anomalous transport. The anomalous particle transport, as well as the correlation between the existence of an instability and its contribution to the transport, is one of the most important issues in understanding the confinement of a magnetized plasma.

The most notable one is that toroidal rotation reverses its direction after a low-mode ( L-mode) to H-mode transition. To explain this phenomenon, one has to deal with the toroidal momentum equation directly. To this end, we adopt the neoclassical quasilinear theory to calculate toroidal stress induced by electrostatic fluctuations and examine its qualitative behavior. Of course, this neoclassical theory must take into account the fact that the presence of magnetic island breaks the toroidal symmetry of a tokamak.

The linear drift kinetic equation with plasma flows as the driving terms is

$$
\begin{aligned}
& \frac{\partial f}{\partial t}+\left(\left(v_{\|}+V_{\|}\right) n+V_{E}\right) \cdot \nabla f-C(f) \\
& =2\left(v / v_{t}\right)^{2}\left(1 / 2-3\left(v_{\|} / v\right)^{2} / 2\right) \\
& \cdot\left(\left((V \cdot \nabla B) / B-(2 / 5) L_{1}^{3 / 2}(q \cdot \nabla B)\right) /(B p)\right) f_{M}
\end{aligned}
$$

where $f$ is the perturbed particle distribution function, $v_{\|}$ is particle speed paralel to $B, v$ is the particle speed, $V_{E}$ is the $E \times B$ drift, $E$ is the electric field, $C$ is the test particle part of the Coulomb collision operator, $V$ is the plasma flow velocity, $q$ is the heat flow, $v_{t}$ is the thermal speed of the particle, $L_{1}^{(3 / 2)}=5 / 2-x^{2}$ is a Laquerre polinomial, $x=v / v_{t}$ and $f_{M}$ is the equilibrium shifted Maxwellian distribution function [19].

In Ohmically heated tokamak plasmas or in RF-heated tokamak plasmas where wave momentum is zero, there is no obvious toroidal momentum source. The steady-state toroidal rotation velocity profile is determined approximately by balancing the diffusion term and the convective term. There are many partial results of the application of drift kinetic equation for different regimes of tokamak plasma confinements.

Many physical dynamical systems are spatially extended and exhibit cooperative wave patterns as well as high-dimensional chaotic attractors. Example for this kind of complex dynamics is interacting spiral waves in excitable media.

Only some model parameters have to be determined from experimental data. Often, however, even the structure of the models is not or only partially known and has to be identified using observed data. Structure identification and parameter estimation, several methods have been suggested based on non-linear regression, state space reconstruction, perturbation or synchronization.

More difficult to identify and to model are structural inhomogeneties which occur due to long-range connections between different(remote) locations in the spatially extended system introducing additional coupling. Correct identification of these long-range links is of crucial importance for correct modeling. A network is balanced if the in-degree of any nodes is equal to its out-degree.

We shall suggest a system identification method based on steady-state stabilization. To stabilize a steady state, we add to control signal $u_{i}$ such that we have controlled network. Then we exploit the dependence of the equilibrium on control parameters. In this way we obtain all relevant information to identify the underlying dynamics as well as coupling structure [20].

We shall consider in the following time-spatially extended systems that can approximately be described by large sets of partial differential equations. We first stabilize a stationary state (at least in some region) and then exploit the dependence of the equilibrium on control parameters. In this way we obtain all relevant information to identify the underlying dynamics as well as coupling structure.

Statistical behavior described by power-laws is appealing to attribute to the existence of "criticality" in the underlying dynamics. In the statistical mechanics sense, this means the proximity of a phase transition, and one of the perminent ideas in this respect is "self-organized criticality" (SOC). This is usually taken to mean that no fine-tunning to a critical point in the classical sense is needed and the system at hand is driven to such a critical state.

The measured signal can be considered after e.g. spatial or temporal thresholding. Example is given by the local particle flux in the perimeter of a fusion device. In particular for classical SOC systems, with uncorrelated driving, global waiting time statistics are described most often by a Poisson process.

Understanding the field lines chaotic structures in stationary flows can be considered as the first step towards understanding transport in these flows. This chaos of field lines has been investigated for a long time in plasma physics, especially when concerned with the conception of magnetically confining devices such as tokamaks.

Potential interest in computing field lines for a flux free field is the possibility to compute Poincare sections of the field lines. We should also be able to discriminate 
the physical importance of islands of regular motion within a stochastic sea in the Poincare section. Let $v$ be a three-dimensional vector field. Field lines of $v$ are curves which are tangent to the field at any point.

The anomalous transport is non-Fickian transport.

The entropy of nonequilibrium stationary states differs from the entropy of local equilibrium states. In some cases the long range correlations of the nonequilibrium state is not captured by the Gibbs-Shannon entropy of local equilibrium states. We can examine the entropy of the stationary nonequilibrium states $\mu_{\alpha, \beta}^{N}$ [21]. We solve the nonlocal-in-time transport equation with a $\psi(s, t)$ containing a power-law dependence in both $s$ (a Levy-like distribution) and $t$, which necessitates the strong $s, t$ coupling. The interplay between two coupled power laws is clearly shown in the changes in the arrival times and dispersion.

We write the drift kinetic equation as

$$
\begin{aligned}
& \frac{\partial f}{\partial t}+\left(\hat{e}_{\|} \cdot v_{\|}+v_{\Delta}+v_{E}\right) \cdot \nabla f-\frac{q}{m} \cdot \nabla_{\|} \cdot \phi \frac{\partial f}{\partial v_{\|}} \\
& -\frac{q}{m} \nabla \phi \cdot\left(\frac{v_{P B}}{B} \frac{\partial f}{\partial \mu}+\frac{v_{k}}{v_{\|}} \frac{\partial f}{\partial v_{\|}}\right)=0
\end{aligned}
$$

where $\mu=v_{\perp}^{2} / 2 B$ is the magnetic moment.

Here the $v_{E} \cdot \nabla f$ term is the $E \times B$ nonlinearity. It dominates and is responsible for the first saturation of an instability. This is true both in fluid and in kinetic systems. The third term is the parallel nonlinearity. It gives nonlinear Landau damping and has sometimes been ignored in kinetic codes. The last term is the nonlinear magnetic drift resonance. It is analogous to nonlinear Landau damping but applies to the perpendicular magnetic drift resonance. It has sometimes been neglected in kinetic codes.

Gyro-Landau fluid (or gyrofluid) equations are an extension of the usual equation to include models of kinetic resonances and gyro-averaging which play important roles in fusion plasma turbulence.

Based on the linearized kinetic drift equation and the fluid equations including the density perturbation term driven by RF waves, the criterion of the destabilization of the poloidal plasma rotation is analytically derived. It is shown that the edge poloidal plasma rotation can be destabilized during electron cyclotron resonant heating (ECRH) in the present RF power level [22].

The production of the poloidal density asymmetry by using ECRH in the collisional plasmas is studied based on the linearized drift kinetic equation:

$$
\frac{\partial f_{0}}{\partial t}+v_{\|} b \cdot \nabla f_{1}+z_{\alpha} e E \cdot b v_{\|} \frac{\partial f_{0}}{\partial W}=C\left(f_{1}\right)+Q\left(f_{0}\right),
$$

where $f_{0}$ and $f_{1}$ are the undisturbed and distorted parts of electron distribution function due to $r f$ waves, $z_{\alpha}$ is the charge number of the particle species $\alpha(\alpha=i, e), E$ is the poloidal electric field, $b$ is direction of magnetic field, $v_{\|}$is the parallel velocity of particle, and $C$ and $Q$ are the linearized Coulomb collision operator and the RF quasilinear operator, correspondingly.

We formulate the criterion of the destabilization of the poloidal plasma rotation during ECRH in the collision plasmas, which is relevant to the edge plasma in tokamaks.

Calculation of the thermodynamic fluctuations can be applied to the large class of the so-called "reactive" instabilities. This class includes unstable modes important for thermonuclear machines, as the ion or electron temperature gradient modes (ITG) or (ETG). The relation between the electrostatic entropy and the Lagrangian description of motion of the high temperature system of Coulomb interacting particles is investigated. We can proceed to the derivation of the equation of the constrained motion according to the Lagrangian. The application of the Hamiltonian's action principle gives

$$
\int_{t_{1}}^{t_{2}} \delta L \mathrm{~d} t=\int_{t_{1}}^{t_{2}} \sum_{n=1}^{N_{p}}\left(\frac{\partial L}{\partial \bar{x}_{n}}-\frac{\mathrm{d}}{\mathrm{d} t} \frac{\partial L}{\partial \bar{v}}\right) \cdot \delta \bar{x}_{n}(t) \mathrm{d} t=0
$$

One obtains the equation of motion for the generic s-particle.

The toroidal axis-symmetric equilibrium is described by the Grad-Shafranov equation

$$
j_{\phi}=\frac{c}{4 \pi} R \nabla \cdot\left(R^{-2} \nabla \psi\right)
$$

The average of the Lagrangian can be expressed as a linear function of the magnetic entropy and of the plasma thermal energy only. $L$ takes on the form

$$
L=U+\frac{m n \Delta V}{3} \tilde{V}^{2} \cdot S_{p}+(\lambda+2) \phi_{\text {int }}
$$

In condition of stationary equilibrium the thermal energy of the electrons is constant in time and the power balance is expressed by the relation

$$
-\int_{\partial \Omega} q_{h} \cdot \mathrm{d} \bar{S}+\int_{\Omega} E_{e x t} \cdot j_{p} \mathrm{~d} \Omega+\int_{\Omega} p_{E} \mathrm{~d} \Omega=0
$$

Here $q_{h}$ is the heat flux related to the thermal conductivity of the electrons and the third therm contains the contribution of the net power density deposited on electrons (auxiliary power, electron-ion energy transfer, non diffusive losses) in addition to the Ohmic contribution described by the second therm.

Given the need for robust variational principles predicting the overall behavior of fusion plasmas, the entropy-based methods are expected to be of increasing importance in this field. The multiregion-relaxation variational approach holds strong promise of being the most satisfactory mathematical fundation on which to 
base the selection of the MHD toroidal equilibrium problem posed by Grad over forty years ago. In the paper [23] is studied an optimal control problem arising in plasma transport which is governed by a singularly perturbed system.

A formulation of the Euler-Lagrange equations was given for problems of the calculus of variations with fractional derivatives. There is no general formulation of a fractional version of Pontryagin's Maximum Principle. With a fractional notion of Pontryagin extremal, one can try to extend it to the more general context of the fractional optimal control [24]. The drift kinetic equation can be derived from full kinetic equation, i.e. Vlasov-Poisson-Fokker-Planck equation.

We have the next theorem.

Theorem. Let us have the fusion reactor problem. Then simulation and optimization of the results could be obtained by the methods of advanced optimal control.

Proof. We consider the control systems of the VlasovPoisson-Fokker-Planck equations

$$
f^{\prime}=A f+B u, u=F f+w, w=F_{1} f+w_{1}
$$

where $A$ is direct sum of operators $A_{i}, i=1,2, \cdots, k$

$$
A_{i} \boldsymbol{f}_{i}=-\left(v \cdot \nabla_{x}\right) \boldsymbol{f}_{i}, B F \boldsymbol{f}=-\nabla_{v}\left(\left(E_{\sigma}-\beta \cdot v\right) \boldsymbol{f}\right)
$$

and $B F_{1} \boldsymbol{f}=\sigma \cdot \nabla_{v} \cdot \nabla_{v} \cdot \boldsymbol{f}$, vector $\boldsymbol{f}$ is direct sum of vectors $\boldsymbol{f}_{i}, i=1,2, \cdots, k$ [25]. $E(t, x)$ is the force field acting on particles. The strictly positive parameters $\beta$ and $\sigma$ model a certain type of interaction between particles.

Consider the following IF-THEN rules:

$$
\begin{aligned}
& \text { IF } m_{(1)} \text { is } M_{i 1} \text { and } \ldots \text { IF } m_{(p)} \text { is } M_{i p} \text { THEN } \\
& (\mathrm{d} f / \mathrm{d} t)=\left(A_{i}+E_{i}\right) f(t)+A_{d i} f(t-h)+B u(t), \\
& i=1,2, \cdots, k
\end{aligned}
$$

where $M_{i j}$ are fuzzy sets and $m_{(1)}, \cdots, m_{(p)}$ are given premise variables, and $E_{i}$ are the uncertain matrices. The fuzzy system is hence given by the sum of equations

$$
\begin{aligned}
& (\mathrm{d} f / \mathrm{d} t)=a_{(i)}\left(A_{i}+E_{i}\right) f(t)+A_{d i} f(t-h)+B u(t), \\
& i=1,2, \cdots, k
\end{aligned}
$$

where $a_{(i)}$ are the fuzzy basis functions [26]. We have the general formalism of optimal control in the presence of noise. Optimal feedback design based on deterministic model is minimization of total fluctuation energy of instabilities, as well as minimization of control power

$$
J\left(t_{f}\right)=\int_{0}^{t_{f}}\left(f_{N}^{T}(t) \cdot Q \cdot f_{N}(t)+u^{T}(t) \cdot R \cdot u(t)\right) \mathrm{d} t
$$

For Lagrangian

$$
\begin{aligned}
& L\left(f_{N}, f_{N}^{\prime}\right)=f_{N}^{T} \cdot Q \cdot f_{N}(t)+u^{T}(t) \cdot R \cdot u(t) \\
& +\lambda^{T}(t)\left(A f_{N}(t)+B(\mu C) u(t)-f_{N}^{\prime}(t)\right)
\end{aligned}
$$

It holds that $\frac{\mathrm{d}}{\mathrm{d} t}\left(\frac{\partial L}{\partial f_{N}^{\prime}}\right)-\frac{\partial L}{\partial f_{N}}=0$ with optimal control

$$
u(t)=-R^{-1}(B(\mu C))^{T} \cdot \lambda(t) .
$$

Here $\mu$ is activation function of a deterministic recurrent ANN. It can be calculated with Paricle in Cell (PIC) method.

The general appropriate reduced model is

$$
f_{N}^{\prime}=A f_{N}+B(\mu C) u+D(\xi F) \psi_{N}
$$

where $D(\xi F) \psi_{N}$ is stochastic noise, and $\xi$ is activation function of a stochastic recurrent ANN [27]. It can be calculated with Monte Carlo method.

The solution under optimal feedback is

$$
f_{N}^{\prime}=\left(A-B K_{c}\right) f_{N}(t)+D(\xi F) \psi_{N}(t)
$$

and

$$
\begin{aligned}
& f_{N}(t)=\phi\left(t, t_{0}\right) \cdot f_{N}\left(t_{0}\right) \\
& +\int_{t_{0}}^{t} \phi(t, \tau) D(\xi F) \psi_{N}(\tau) \mathrm{d} \tau
\end{aligned}
$$

On this way the theorem is shown.

As actuators in the process we can use also NBI, ICRF, ECCD and it must be used in such a way that the optimal result is obtained.

We considered the following VPFP system with neural networks with delays

$$
\begin{aligned}
& \frac{\partial f_{i}}{\partial t}=A f_{i}+B\left(\mu_{i} C\right) u+\sum_{j=1}^{\infty} w_{i j} q_{j} f_{i}(t, x, v) \\
& +\sum_{j=1}^{\infty} h_{i j} l_{j}\left(f_{i}\left(t-\tau_{j}(t), x, v\right)\right)+J_{i}
\end{aligned}
$$

where $f=\left(f_{1}, f_{2}, \cdots, f_{k}\right), \quad f_{i}(t, x, v)$ is the state of the plasma at time $t, l_{j}$ denotes the activation function of the $i$ th neuron at time $t-\tau_{j}(t)$. On the synchronization problems of neural networks, we see that there have been some research work [28]. We want to obtain and adaptive synchronization controller to achieve exponential synchronization for a class of VPFP systems. Here $w_{i j}$ denotes the strength of the $j$ th neuron on the $i$ th neuron at time $t$ and in the space $(x, v), h_{i j}$ strength of the $j$ th neuron on the ith neuron at time $t-\tau_{j}(t)$ and in the space $(x, v), \tau_{j}(t)$ are time-varying delays of neural networks.

The "self-organized criticality" (SOC) is usually taken to mean that the system is driven to a critical state. We introduce the following. The entropy of fuzzy dynamical system has been defined. Let $P=\{A(1), A(2), \cdots, A(n)\}$ be a measurable fuzzy partition of $X$. The entropy $H(P)$ of $P$ is defined as $H(P)$ is a sum of $\mathrm{n}$ elements of the type: $-m(A(i)) \log m(A(i))$. The entropy of fuzzy dynamical system $(X, F, m, T)$ is the number 
$h(T)$ defined as $h(T)=\sup H(P, T)$, where the supremum is taken over all finite measurable fuzzy partitions of $X$ and $T: X$ to $X$ is a fuzzy measure-preserving transformation. $H(P, T)$ is a limit, as $n$ tends to infinity, of the expression $H$ over span of potentions of $T$ multilied by $P$ and then divided by $n$. The limit $H(P, T)$ exists for each fuzzy process with finite measure. The entropy $H(P, T)$ was introduced by Kolmogorov and Sinai and is often referred to as the Kolmogorov-Sinai invariant. The Kolmogorov-Sinai invariant was the first invariant which was not a spectral nature. In the deterministic case in equilibrium state the minimum of fuzzy Kolmogorov-Sinai entropy must be obtained. If we consider the stochastic case then in equilibrium the maximum of fuzzy Jaynes entropy must be obtained [29].

In the case of plasma confinement there are often only experimental obtained data that are not calculated by formulas. It is the case when disruption is possible. In these cases we must use the method of artificial neural networks for describing the problem [30]. After implementation of artificial neural networks our advanced variational calculus can be used to find the optimal path of plasma transport without disruption.

\section{Conclusion}

The advanced optimal control of fusion plasma confinement is solved by the methods of artificial intelligence and drift kinetic equations. It means that fuzzy systems and artificial neural networks should be applied in the process of optimization. On such a way the using of neutral beam injection, ion-cyclotron range of frequencies and electron cyclotron current drive can be analysed as actuators together with Vlasov-Poisson-FokkerPlanck equations. We construct an asymptotic solution of the equation of magnetohydrodynamics (MHD) at high Reynolds numbers. One of the quantities that may be considered in this context is the waiting time of an experimental or observational parameter. As numerical tools we can use fuzzy, adaptive and local Monte Carlo methods or particle in cell method via advanced variational calculus.

\section{REFERENCES}

[1] G. Zaslavsky, R. Z. Sagdeev, D. A. Usikov and A. A. Chernikov, "Weak Chaos and Quasi-Regular Patterns," Cambridge University Press, Cambridge, 1991. doi:10.1017/CBO9780511599996

[2] X. Gao, S. Zhong and F. Gao, "Exponential Synchronization of Neural Networks with Time-Varying Delays," Nonlinear Analysis, Vol. 71, No. 5, 2009, pp. 2003-2011. doi:10.1016/j.na.2009.01.243

[3] X. D. Li, "Existence and Global Exponential Stability of
Periodic Solution for Delayed Neural Networks with Impulsive and Stochastic Effects," Neurocomputing, Vol. 73, No. 4-6, 2010, pp. 749-758. doi:10.1016/j.neucom.2009.10.016

[4] L. Frassinetti, K. E. J. Olofsson, P. R. Brunsell and J. R. Drake, "Resonant Magnetic Perturbation Effect on Tearing Mode Dynamics," Nuclear Fusion, Vol. 50, No. 3, 2010, Article ID: 035005. doi:10.1088/0029-5515/50/3/035005

[5] E. Minardi, "Thermodynamics of High Temperature Plasmas," Entropy, Vol. 11, No. 1, 2009, pp. 124-221. doi:10.3390/e11010124

[6] E. Schuster, J. Dalessio, M. L. Walker, Y. In and J. S. Kim, "Extending the RWM Stability Region by Optimal Feedback Control," 47th IEEE Conference on Decision and Control, Cancun, 9-11 December 2008, pp. 31453150 .

[7] X. Garbet, Y. Idomura, L. Villard and T. H. Watanabe, "Gyrokinetic Simulations of Turbulent Transport," Nuclear Fusion, Vol. 50, No. 4, 2010, Article ID: 043002.

doi:10.1088/0029-5515/50/4/043002

[8] G. Van Oost, V. V. Bulanin, A. J. H. Donne, et al., "Multi-Machine Studies of the Role of Turbulence and Electric Fields in the Establishment of Improved Confinement in Tokamak Plasmas," Plasma Physics and Controlled Fusion, Vol. 49, 2007, pp. A29-A44. doi:10.1088/0741-3335/49/5A/S03

[9] B. P. Milligen, B. A. Carreras, V. Antono, et al., "LongRange Correlation Analysis of Plasma Turbulence," 26th EPS Conference on Controlled Fusion and Plasma Physics, Vol. 23J, 1999, pp. 49-52.

[10] D. Rastovic, "Fixed Point Theorem and Infinite Fuzzy Logic Controllers," Cybernetica, Vol. 39, No. 1, 1996, pp. 49-51.

[11] H. Emamirad, "Scattering Theory for Linearized Boltzmann Equation," Transport Theory and Statistical Physics, Vol. 16, 1987, pp. 503-528. doi: $10.1080 / 00411458708204304$

[12] D. Rastovic, "Feedback Stabilization of Some Classes of Nonlinear Transport Systems," Rendiconti del Circolo Matematico di Palermo, Vol. 51, No. 2, 2002, pp. 325332. doi:10.1007/BF02871658

[13] D. Rastovic, "Vlasov-Poisson-Fokker-Planck Equations and Stabilization System," Analele Universitatii de Vest din Timisoara, Vol. 42, No. 1, 2004, pp. 141-148.

[14] C. S. Rodrigues, A. P. S. Moura and C. Grebogi, "Random Fluctuation Leads to Forbidden Escape of Particles," Physical Review E, Vol. 82, No. 2, 2010, Article ID: 026211. doi:10.1103/PhysRevE.82.026211

[15] D. Rastovic, "A Note on Stability Properties of Integrated Semigroups," Acta Mathematica et Informatica Universitatis Ostraviensis, Vol. 3, No. 1, 1995, pp. 61-65.

[16] Y. Sarazin, V. Grandgirard, J. Abiteboul, et al., "Large Scale Dynamics in Flux Driven Gyrokinetic Turbulence," Nuclear Fusion, Vol. 50, No. 5, 2010, Article ID: 054004. doi:10.1088/0029-5515/50/5/054004

[17] G. A. Ratta, J. Vega, A. Murari, et al., "An Advanced Disruption Predictor for JET Tested in a Simulated RealTime Environment," Nuclear Fusion, Vol. 50, No. 5, 2010, 
Article ID: 025005.

[18] D. Rastovic, "Fractional Fokker-Planck and Artificial Neural Networks," Journal of Fusion Energy, Vol. 27, No. 3, 2008, pp. 182-187. doi:10.1007/s10894-007-9127-9

[19] K. C. Shaing, "Toroidal Momentum Confinement in Neoclassical Quasilinear Theory in Tokamaks," Physics of Plasmas, Vol. 8, No. 1, 2001, pp. 193-200. doi:10.1063/1.1332986

[20] D. Yu and U. Parlitz, "Inferring Local Dynamics and Connectivity of Spatially Extended Systems with LongRange Links Based on Steady-State Stabilization," Physical Review E, Vol. 82, No. 2, 2010, Article ID: 026108.

[21] C. Bernardin and C. Landim, "Entropy of Stationary Nonequilibrium Measures of Boundary Driven Symmetric Simple Exclusion Processes," Journal of Statistical Physics, Vol. 141, No. 6, 2010, pp. 1014-1038. doi:10.1007/s10955-010-0082-X

[22] C.-G. Liu and S.-J. Qian, "Poloidal Flow Destabilized by Electron Cyclotron Resonant Heating in Collision Tokamak Plasmas," Chinese Physics Letters, Vol. 15, No. 1, 1998, p. 35. doi:10.1088/0256-307X/15/1/014

[23] R. L. Dewar, M. J. Hole, M. Mc Gann, et al., "Relaxed Plasma Equilibria and Entropy-Related Plasma SelfOrganization Principles," Entropy, Vol. 10, No. 4, 2008, pp. 621-634. doi:10.3390/e10040621

[24] D. Rastovic, "Fractional Variational Problems and Particle in Cell Gyrokinetic Simulations with Fuzzy Logic Approach for Tokamaks," Nuclear Technology \& Radiation Protec- tion, Vol. 24, No. 2, 2009, pp. 138-144. doi:10.2298/NTRP0902138R

[25] D. Rastovic, "Optimal Control of Tokamak and Stellarator Plasma Behaviour," Chaos, Solitons \& Fractals, Vol. 32, No. 2, 2007, pp. 676-681. doi:10.1016/j.chaos.2005.11.016

[26] D. Rastovic, "Fuzzy Scaling and Stability of Tokamaks," Journal of Fusion Energy, Vol. 28, No. 1, 2009, pp. 101106. doi:10.1007/s10894-008-9154-1

[27] D. Rastovic, "Targeting and Synchronization at Tokamak with Recurrent Artificial Neural Networks," Neural Computing \& Applications, Vol. 21, No. 5, 2012, pp. 10651069. doi:10.1007/s00521-011-0527-4

[28] K. Wang and Z. D. Teng and H. J. Jiang, "Global Exponential Synchronization in Delayed Reaction-Diffusion Cellular Neural Networks with the Dirichlet Boundary Conditions," Mathematical and Computer Modelling, Vol. 52, No. 1-2, 2010, pp. 12-24. doi:10.1016/j.mcm.2009.05.038

[29] D. Rastovic, "Applications of Artificial Intelligence and Multi-Variable Control of Chaos on Tokamak Equilibriums," In: Glow Discharges and Tokamaks, Nova Science Publishers, Inc., 2011, pp. 407-418.

[30] M. Camplani, B. Cannas, A. Fanni, et al., "Tracking of the Plasma States in a Nuclear Fusion Device Using SOMs," Neural Computing \& Applications, Vol. 43, 2009, pp. $430-437$. 